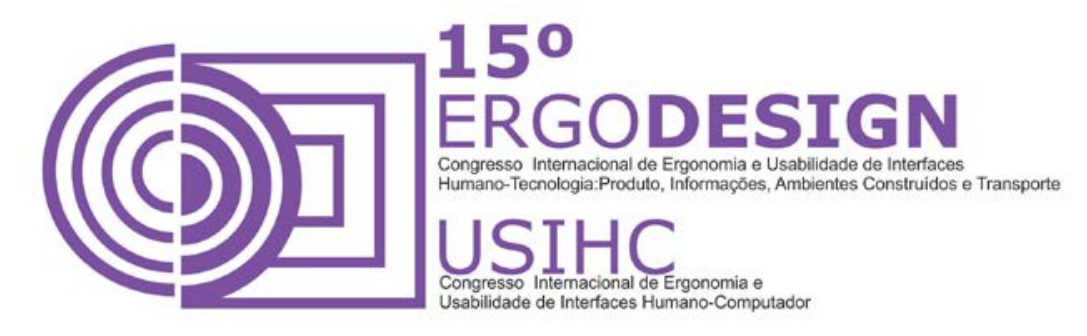

\title{
O CONHECIMENTO DA CULTURA PARA A CRIAÇÃO DE SISTEMAS INTELIGENTES, UM ESTUDO DAS NOTÍ́CIAS EM MÍDIAS DIGITAIS
}

\section{KNOWLEDGE OF CULTURE TO CREATE SYSTEM THINKING, A STUDY OF NEWS MEDIA DIGITAL}

\author{
NUNES, Juliana (1); \\ QUARESMA, Manuela (2)
}

(1) Laboratório de Ergodesign e Usabilidade de Interfaces da PUC-Rio (LEUI), Mestranda em Design, Bolsista CNPq

e-mail:julinunesb@gmail.com

(2) Laboratório de Ergodesign e Usabilidade de Interfaces da PUC-Rio (LEUI),

Doutora em Design

e-mail:mquaresma@puc-rio.br

\begin{abstract}
RESUMO
As novas tecnologias digitais aliadas à web mudaram a maneira pelas quais as pessoas se relacionam com as mídias veiculadoras de notícias. Isso se torna um desafio para quem trabalha com a manipulação de conteúdo jornalístico, porque existe uma grande resistência em se mudar uma cultura sedimentada. Portanto, para se criar sistemas melhores é preciso começar mapeando a cultura, entendendo-a como todas as relações interpessoais que auxiliam na criação de sistemas. Esse artigo objetiva apresentar 0 posicionamento de profissionais de mídia sobre alguns questionamentos recentes pelos quais as redações de jornais vêm enfrentando através da aplicação da técnica Delphi.
\end{abstract}

Palavras-chave: Mapeamento da Cultura; Delphi; Notícias; Mídias Digitais.

\section{ABSTRACT}

The new digital technologies allied to web changed the way in which people relate to news media. This becomes a challenge for who works with manipulation of journalistic content because there is a great resistance to change a settled culture. Therefore, to create better systems it's necessary to start mapping the culture, understanding it as all interpersonal relationships that assist in creating systems. This paper 


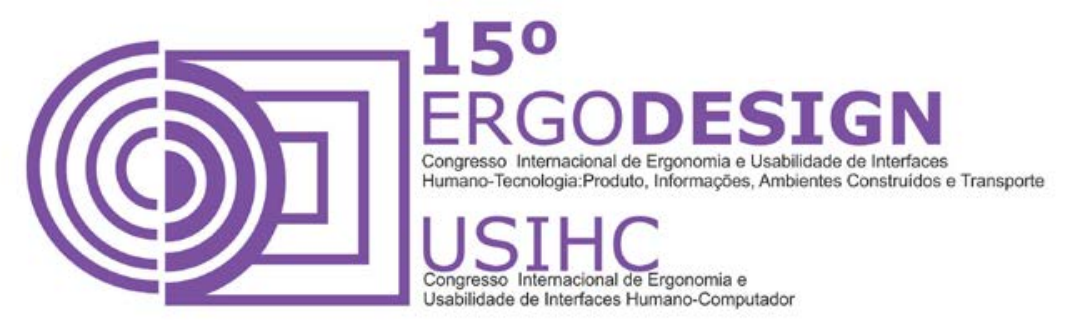

presents media professionals positioning on some recent questions for which the newspaper offices are facing through the application of the Delphi technique.

Keywords: Culture Mapping, Delphi, News, Digital Media, System Thinking.

\section{INTRODUÇÃO}

Os sistemas inteligentes surgiram como uma crítica ao reducionismo, formalizado por René Descartes no século 17. O reducionismo é a ideia que qualquer sistema pode ser entendido estudando suas partes e, nesse entendimento, pode-se pressupor que o todo é igual à soma de suas partes. Mas para os sistemas inteligentes, o todo é maior do que a soma das suas partes porque se baseiam no entendimento que o mundo é sistêmico e o todo não pode ser totalmente compreendido somente pelas suas partes constituintes confiando, assim, na síntese para ver o todo e a interação entre as partes. (FLOOD, 2010; MORVILLE, 2014)

É um sistema de difícil concepção porque nossa sociedade está organizada pelo princípio oposto. O reducionismo e a especialização tiveram tanto êxito na Revolução Industrial e no avanço científico, que foram incorporados na nossa cultura. Por exemplo, nas escolas divide-se as matérias em conhecimentos e os estudantes por graduações. Não é que o sistema reducionista esteja errado, mas nem tudo pode ser resolvido em partes. Um bom exemplo de sistema inteligente pode ser compreendido pela Biologia. Um organismo não pode ser amplamente apreendido somente pelo comportamento de algumas partes, pois estas são interrelacionadas e influenciam umas às outras. Portanto, um organismo se comporta como "mais do que a soma de suas partes." (FLOOD, 2010; MORVILLE, 2014)

Um dos primeiros passos para um projeto bem sucedido de sistemas inteligentes é aquele que consegue mapear e compreender tanto a cultura da companhia quanto a cultura dos seus consumidores para que se adeque aos dois pólos, como demonstra a figura 1.

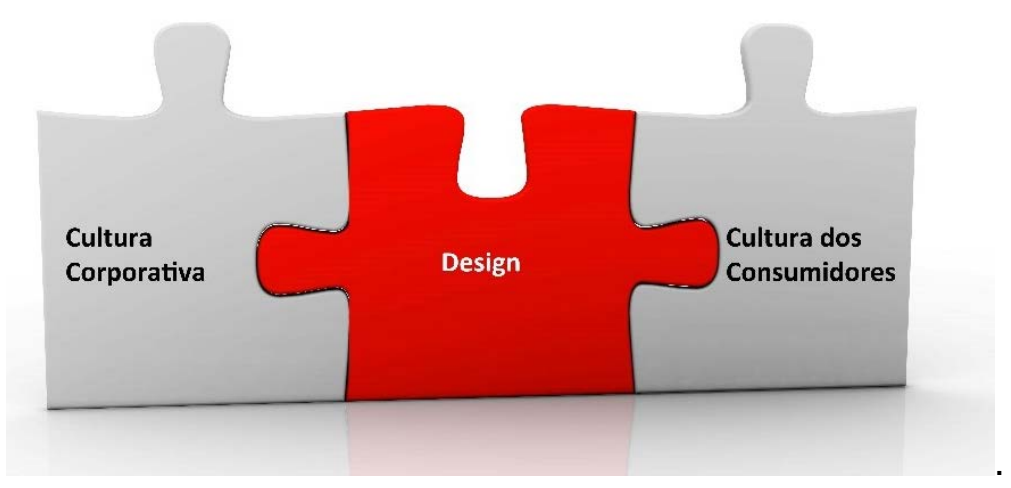

Figura 1: projetar para se ajustar a uma cultura bilateral. (imagem adaptada. MORVILLE, 2014) 


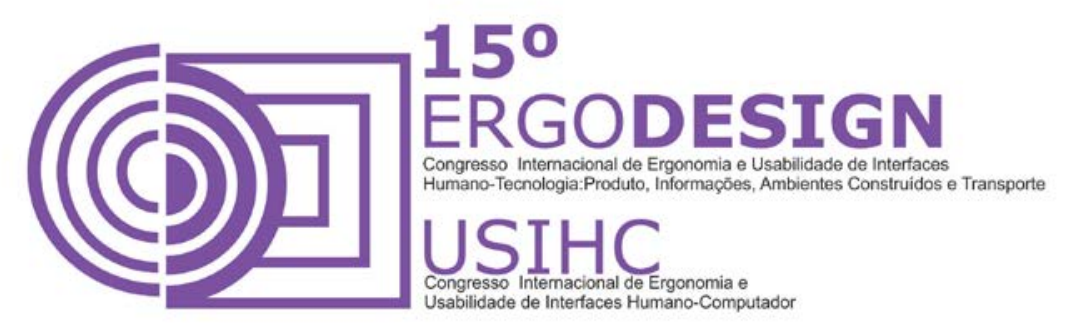

Cultura é um poderoso, quase um inconsciente, conjunto de forças que moldam nossos comportamentos individuais e coletivos. E para entender qualquer cultura, é preciso estudar seu contexto. (MORVILLE, 2014)

Percebe-se que muitas empresas estão encontrando dificuldades em cobrar pelos conteúdos disponibilizados na rede. No que diz respeito às notícias, muitos portais as veiculam gratuitamente, mas o material gratuito também tem o seu preço. Atualmente vimos sucumbir jornais, livrarias, bibliotecas e privacidade. Agora procuramos por respostas no mar de propagandas, pensando cuidadosamente (ou não) sobre onde olhar, em quem confiar e em que acreditar. (MORVILLE, 2014, livro digital, tradução nossa)

Uma das possibilidades de entendimento do que está acontecendo com a indústria jornalística brasileira é mapear a cultura de quem está familiarizado com o funcionamento de uma redação de jornal. Esse artigo pretende contribuir com uma pesquisa realizada com profissionais que produzem ou veiculam notícias jornalísticas ou que tenham alguma familiaridade com uma redação de jornal sobre como as notícias diárias são apresentadas atualmente e quais os principais desafios da indústria jornalística. A técnica Delphi foi a selecionada para a coleta dos dados desses profissionais.

\section{A TÉCNICA DELPHI}

Essa técnica pode ser utilizada quando uma informação acurada é indisponível ou dispendiosa para se obter ou os modelos de avaliação requerem inputs subjetivos ao grau que estes se tornam os parâmetros dominantes (LINSTONE e TUROFF, 1975). Visa reduzir o grau de incertezas e à resposta de problemas complexos utilizando como mecanismo para tal o consenso por meio da opinião de especialistas (EVERLING, 2011). Uma das grandes vantagens é eliminar aspectos de autodefesa e de competição que geralmente são obstáculos quando se tenta obter o consenso de um grupo. (FRISONI, 2000)

Ainda que a palavra escrita permita um conteúdo emocional, o processo da Delphi tende a minimizar os sentimentos e informações normalmente comunicado de algumas maneiras, como o tom de voz, o gesto das mãos ou uma expressão facial (LINSTONE e TUROFF, 1975). Podese evidenciar esse fator como uma limitação da técnica, já que em muitas instâncias essas manifestações são partes altamente informativas no processo de comunicação.

Para Candido et al. (2007 apud EVERLING, 2011), alguns cuidados importantes devem ser tomados: (1) deixar claros os objetivos da pesquisa aos especialistas; (2) evidenciar o tempo necessário para participar; (3) retornar os resultados da rodada anterior aos especialistas; (4) evitar o excesso de rodadas procurando limitá-las no máximo a quatro; (5) evitar questões que tenham mais do que um questionamento; (6) redigir as perguntas com objetividade para evitar a subjetividade da sua interpretação pelos especialistas; (7) estruturar perguntas que conduzam para uma resposta direta. 


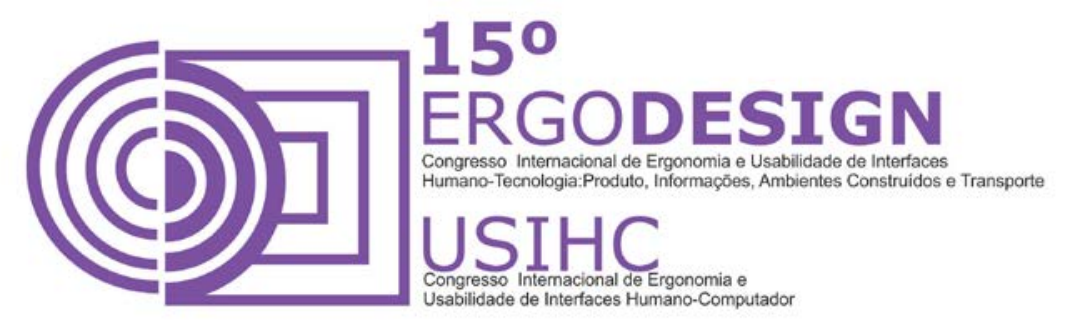

Geralmente a Delphi passa por quarto fases distintas. A primeira fase é caracterizada pela exploração de um assunto sob discussão, onde cada indivíduo contribui adicionando informação que acredita ser pertinente ao assunto. A segunda fase envolve o processo de atingir um entendimento de como o grupo concebe o assunto. Se há uma discordância muito grande, então esta é explorada na terceira fase para trazer à tona as razões subjacentes para as diferenças. A última fase, uma avaliação final, ocorre quando a informação recolhida anteriormente foi inicialmente analisada e as avaliações foram realimentadas para apreciação. (LINSTONE e TUROFF, 1975)

\section{DESCRIÇÃO DO PROCEDIMENTO APLICADO}

O objetivo de aplicar a técnica Delphi foi o de obtenção da opinião de profissionais que produzem ou veiculam notícias jornalísticas ou que tenham alguma familiaridade com uma redação de jornal sobre como as notícias diárias são apresentadas atualmente e quais os principais desafios da indústria jornalística.

Para essa pesquisa foram elaboradas 4 perguntas, sendo 3 abertas e 1 de escala de avaliação. O objetivo das perguntas abertas foi de proporcionar aos especialistas a exposição livre de suas opiniões sem restrições. Já na escala de avaliação, diferentemente, a participação dos respondentes limitou-se à atribuição avaliação aos itens apresentados. Assim sendo, pôde-se obter dois tipos de respostas, algumas mais abrangentes e outras mais direcionadas. A seguir as perguntas formuladas na ordem em que foram apresentadas aos participantes:

1) Quando éramos simplesmente um jornal impresso, esse foco singular fazia sentido. Mas temos agora malabarismos na impressão, a web, os aplicativos, os boletins informativos, as mídias sociais, vídeo, uma edição internacional e uma gama de produtos singulares (THE NEW YORK TIMES, 2013)

Contextualizando a afirmação do The New York Times, que se refere à passagem do jornal impresso ao jornal digital, quais são hoje, na sua opinião, os maiores desafios em se fazer um jornal digital diário?

2) Só para citar alguns exemplos, no site do Estadão, o leitor pode opinar sobre uma notícia e no site do G1 o leitor pode escrever sua própria notícia. Ele ainda pode compartilhá-las nas mídias sociais. Qual a sua opinião sobre essa relação de parceria e colaboração que se estabeleceu entre o jornal e o leitor?

3) Considerando os dispositivos: computador pessoal, smartphone e tablet, você acha que o conteúdo jornalístico deve ser IGUAL, DIFERENTE ou COMPLEMENTAR nos 3 dispositivos? Por quê?

4) De acordo com a legenda a seguir, onde:1 = importantíssimo; 2 = muito importante; $3=$ indiferente; 4 = pouco importante; 5 = não é importante e 6 = não sei opinar,

pontue cada veículo indicado a seguir com um número que melhor traduz o grau de importância para veiculação de notícia jornalística:

Facebook, Blogs/Microblogs, Twitter, Youtube, Instagram, Pinterest, Google + 


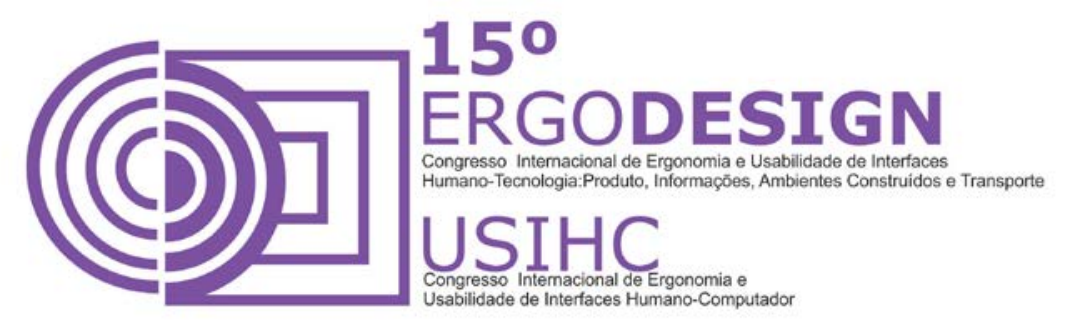

Definiu-se como critério que seriam realizadas duas fases, pois avaliou-se que a coleta dos dados seria suficiente para o objetivo da pesquisa. O veículo de comunicação foi o correio eletrônico e as perguntas encaminhadas a profissionais ligados à prática do jornalismo. Sendo assim, além de jornalistas, procurou-se por áreas afins, como designers que trabalham em jornais e assessores de imprensa. As perguntas foram encaminhadas para 20 profissionais sendo que o retorno dentro do prazo proposto de 10 dias e após outra tentativa por email, obtiveram-se 11 respostas, encerrando-se assim a primeira fase.

A fim de facilitar a comunicação entre os participantes e o pesquisador, na $1^{\mathrm{a}}$. fase as 4 perguntas foram encaminhadas no corpo do email permitindo a aplicação da técnica com maior agilidade dentro do prazo proposto. Junto às perguntas, seguiu um texto explicativo do procedimento e dos objetivos da pesquisa.

Para a $2^{\mathrm{a}}$. fase da técnica elaborou-se um formulário online. Um novo texto explicativo dessa fase foi encaminhado por email e ao final deste, um link redirecionava para um formulário online. As respostas da primeira rodada foram agrupadas por pergunta, sem que os respondentes fossem identificados, criando-se uma página para cada pergunta. Para 0 anonimato dos participantes adotou-se o critério de identifica-los por letra. Para essa rodada final, só entraram as 3 perguntas abertas. Os 11 respondentes puderam avaliar todos os dados reunidos e emitiram por escrito no formulário uma nova resposta ratificando, alterando ou complementando suas respostas anteriores. Ao final do processo, 10 especialistas responderam à segunda fase.

Os resultados obtidos pelos questionários de cada fase foram agrupados em um único documento a fim de facilitar a análise documental gerando o corpus $^{1}$ da pesquisa. Iniciou-se 0 processo com a leitura flutuante ${ }^{2}$ para posteriormente agrupar informações similares relevantes originando, assim, as unidades de registro ${ }^{3}$.

\section{RESULTADOS DA ANÁLISE DE CONTEÚDO}

1) Em relação aos novos desafios de se produzir um jornal diário.

Interessante observar que não houveram pontos divergentes, e sim, vários pontos de vista e muitas respostas complementares. Abaixo seguem os destaques principais das respostas.

${ }^{1}$ Conjunto dos documentos tidos em conta para serem submetidos aos procedimentos analíticos. (BARDIM, 2010, p. 122 apud EARLING, 2011)

${ }^{2}$ Actividade que consiste em estabelecer contacto com os documentos a analisar e em conhecer o texto deixando-se invadir por impressões e orientações. (BARDIM, 2010, p. 122 apud EARLING, 2011)

${ }^{3}$ Unidade de significação e codificação e corresponde ao segmento de conteúdo a considerar como unidade de base, visando a contagem e análise frequencial. (BARDIM, 2010, p. 130 apud EARLING, 2011) 


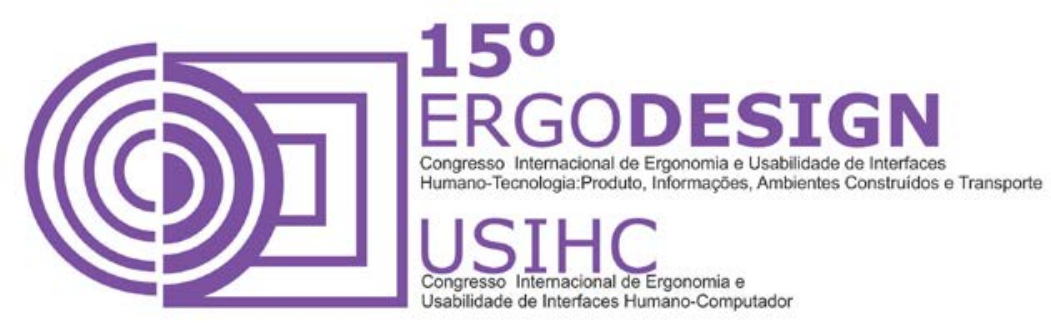

- Novos modelos de negócios

Respondente G - A seção de classificados, por exemplo, respondia por mais de um terço das receitas dos grandes jornais do país.

Com a perda desse tipo de receita, a maioria dos respondentes foi categórica em dizer que o jornal precisa se reinventar como negócio criando novos modelos para geração de receitas. $O$ editor de um grande jornal (respondente F) destaca perguntas importantes que precisam ser consideradas para a criação de novos modelos de negócios:

Deve-se ou não cobrar pelo acesso às informações? E qual a forma mais apropriada de fazer essa cobrança sem que se perca leitores? Como é possível manter os valores de anúncios publicitários que costumam ser cobrados no papel, onde sempre houve pouca concorrência, num ambiente digital, onde a concorrência é gigante? Como equacionar tudo isso para que ainda se possa investir em grandes reportagens, correspondentes no exterior e bons profissionais?

- Concorrência

Respondente $\mathrm{J}$ - Antes, para se informar, as pessoas esperavam pelo jornal da tv, torciam para ouvir o rádio na hora certa, e compravam o jornal no dia seguinte. Hoje, com a web, as fontes de notícias são infinitas e não se limitam apenas às grandes empresas de mídias.

Hoje em dia, uma notícia é veiculada no momento em ocorre o fato e é divulgada em diversas mídias, muitas vezes gratuitamente, o que desencadeia uma concorrência feroz, como destaca o respondente $\mathrm{G}$. O respondente ainda elucida o ponto da gratuidade das informações como um importante ponto para tornar a concorrência com os veículos tradicionais injusta:

Respondente G - Após investir na formação de profissionais e divulgar as reportagens, as mesmas ficam disponíveis na internet e 'todo mundo' pode usá-las sem pagar nada.

A respondente $\mathrm{J}$ destaca também dois pontos fundamentais que acirram a concorrência entre as mídias veiculadoras de notícias - As mídias sociais se tornaram aliadas, mas também concorrentes das mídias tradicionais. Hoje em dia, não é fácil correr atrás do imediatismo da notícia. [...] Competir com essa necessidade de furo, não só dos concorrentes midiáticos, como também de toda uma rede engajada nas redes sociais, tornou-se um grande desafio.

- Jornal diário?

Muitos participantes concordam que a atualização rápida das informações mudou a dinâmica com que as notícias são veiculadas e esse termo "jornal diário" perde completamente o sentido.

Respondente D - O jornal digital, tem que antes de tudo, deixar de ser jornal. O significado não é mais da periodicidade diária.

Respondente $\mathrm{F}$ - O termo diário acaba perdendo o sentido frente à natureza do meio digital.

Respondente $\mathrm{J}$ - O jornalismo tal qual conhecemos não existe mais.

- Jornal impresso 


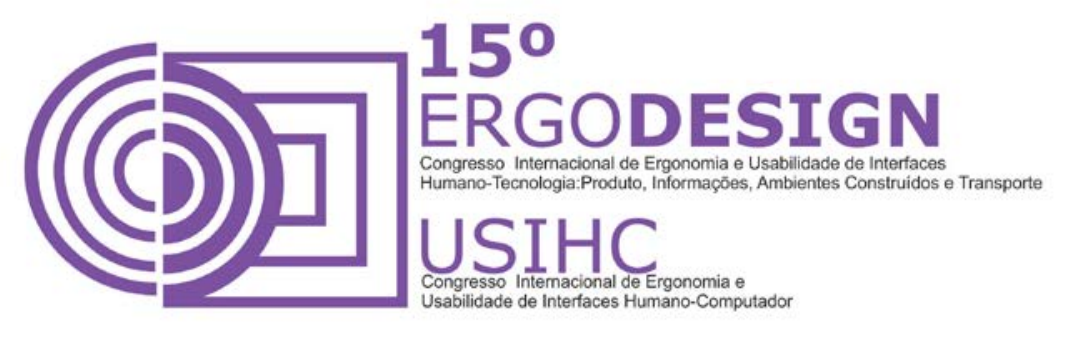

Dois respondentes fizeram menção sobre como o jornal impresso deveria agir para continuar sobrevivendo. A respondente $\mathrm{J}$ concorda com a respondente $\mathrm{H}$ na opinião em que $-[. .$.$] jornais$ diários impressos deveriam apostar no aprofundamento da notícia, a notícia vertical, que o leitor não encontra na web.

O que é interessante destacar nesse comentário é que o jornal digital, na opinião das respondentes, não oferece o aprofundamento de uma notícia, o que levanta alguns questionamentos que deveriam servir para estudos futuros: o jornal digital realmente não oferece a notícia vertical ou não conseguem encontrá-la? Não oferece por que não há interesse do leitor ou porque não há interesse do jornal?

- O usuário e a notícia

Respondente C - É preciso entender os consumidores/usuários, seus comportamentos e suas necessidades e a partir disso criar modelos de negócios e produtos que se adequem a eles. [...] Inovar com foco no usuário.

A colocação do respondente $C$, designer UX, foi de extrema relevância e em consonância com o que foi colocado por Morville (2014), que para a construção de sistemas melhores é preciso o mapeamento das culturas envolvidas. Provavelmente, um caminho factível para a construção de um novo modelo de negócio viável, seja através do entendimento de como os usuários estão consumindo as notícias.

Respondente A - O desafio do digital também acho que seja a forma como as pessoas interagem com a notícia.

2) Em relação à participação dos leitores nos jornais

Alguns questionamentos estão atrelados a essa pergunta, como a democratização da informação e a necessidade de apuração ou não de tudo o que for veiculado.

8 dos 10 participantes concordam que essa é uma relação positiva, porém 6 dos 10 acreditam que deva ocorrer a apuração dos fatos antes da publicação da notícia. Os depoimentos justificam essa aceitabilidade.

Respondente B - Acho que é inevitável e necessária. [...] Não adianta concorrer com este tipo de manifestação [...] é trabalho do profissional do jornalismo verificar e analisar as informações geradas pela "sabedoria das massas."

Respondente C - Não acho que uma notícia criada por um leitor vá tirar o mérito de um jornalista.

Respondente $\mathrm{F}$ - Os leitores naturalmente ganham mais poder no mundo digital. E, quando sites ou jornais digitais resolvem dar espaço em suas páginas [...] eles apenas estão reconhecendo essa mudança.

Respondente G - Essa é uma solução interessante e inevitável. E, ao meu ver, os jornais aproveitam pouco essa oportunidade. [...] Um ponto positivo é que os jornalistas perderam o 'privilégio' de decretar o que é 'notícia' ou não. 


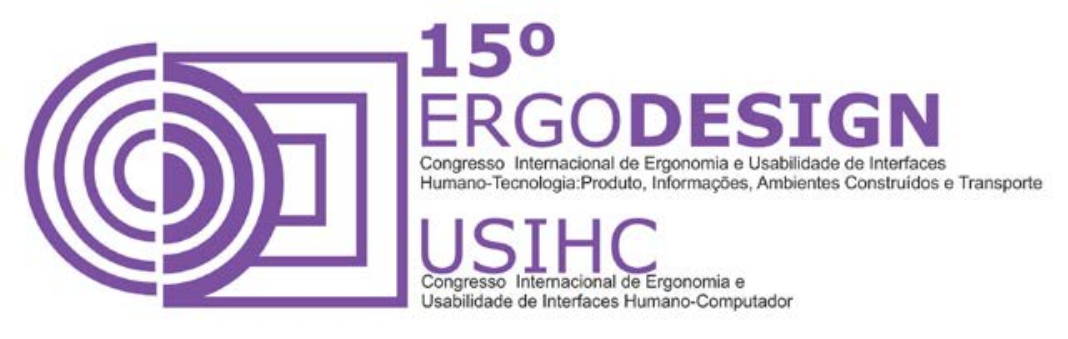

Respondente J - Com uma câmera de celular na mão, todos são potencialmente fontes de notícia. Por que não usar isso a seu favor? Mas isso não tira do jornalista que recebe o furo a filtragem e a necessidade de apuração.

Os 2 respondentes que não concordam que os leitores devam produzir notícia justificam suas opiniões:

Respondente A - Acredito que o que o leitor escreva no site de notícias não deveria ser considerado notícia. Na verdade, acredito que não deveria nem estar junto às notícias.

Respondente $\mathrm{H}$ - Acho que o leitor pode dar sua visão do fato, mas não considero correto dizer que isso seja uma notícia.

3) Se a notícia deve ser IGUAL, DIFERENTE ou COMPLEMENTAR nos dispositivos móveis.

As respostas foram bastante divergentes. Apesar de 6 dos 10 respondentes acreditarem que deva ser igual, a justificativa para tal variou bastante. Em consonância com a linha de pensamento desse artigo, a resposta $F$ traduz bem essa variação das respostas. Os profissionais ainda não sabem como são os novos hábitos de visualização das notícias.

Respondente F - Resposta para essa pergunta não é simples, porque depende muito dos hábitos das pessoas. Não sei dizer bem como as pessoas usam seus gadgets, se elas dividem seu tempo entre telefones, tablets e computadores, ou se há uma concentração em algum desses mecanismos. [...] Nesse novo mundo, cabe à empresa jornalística levar a notícia até uma pessoa, não a pessoa ficar procurando a notícia em vários cantos.

4) Grau de importância das mídias não tradicionais sobre veiculação de notícias jornalísticas (figura 2) 

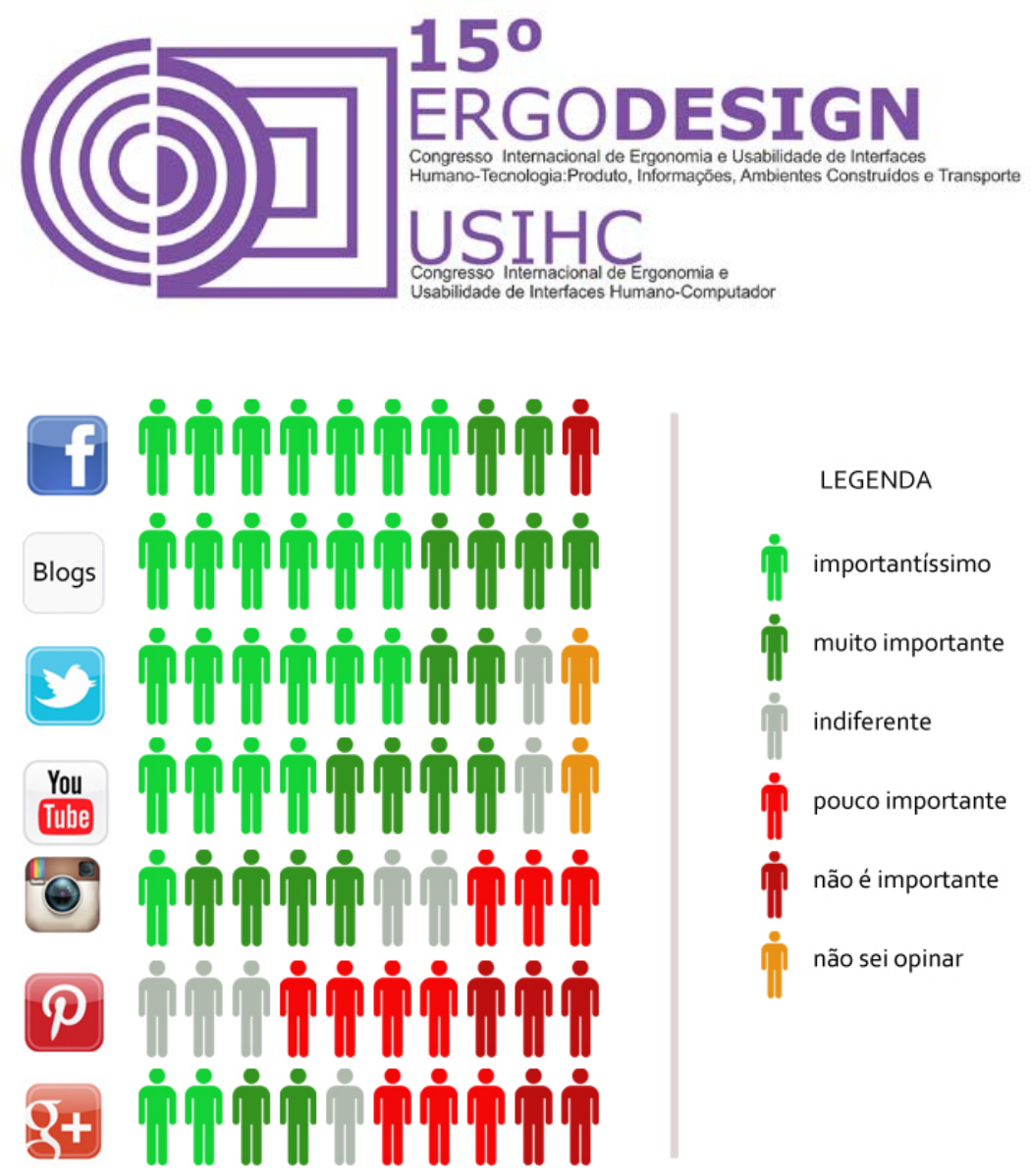

Figura 2: representação do resultado obtido com os dados da pergunta 4

Percebe-se o Facebook, Blogs/Microblogs, Twitter e Youtube como mídias importantes para a veiculação de notícias. As mídias sociais ganham um espaço muito significativo para a veiculação de informação.

\section{CONCLUSÃO}

As transformações pelas quais a indústria jornalística vem passando envolvem mudanças profundas na sua estrutura. Gerenciar conteúdos em multicanais integrando produtos e serviços requer projetar para sistemas integrados. Nesse novo modelo de negócio, os sistemas inteligentes parecem ser uma solução factível. Projetar pensando que as relações entre os produtos e serviços estão inter-relacionados e influenciam uns aos outros, faz com que os profissionais visualizem o projeto como um todo e não fracionado em partes. Portanto, 0 sistema se comporta como "mais do que a soma de suas partes", pois é um organismo que só funciona se a inter-relação entre as partes efetivamente se comunicarem.

Um bom começo para se projetar sistemas melhores é mapear a cultura das partes envolvidas. No caso dos jornais digitais, esse artigo se propôs a analisar a opinião de quem produz, veicula ou está de alguma forma envolvido com a transmissão de uma notícia jornalística. Apesar da pequena mostra coletada através da técnica Delphi, as respostas dos profissionais foram elucidativas para alguns processos pelos quais a indústria jornalística vem enfrentando. Segundo a opinião dos participantes da pesquisa é imperativo que as empresas adotem novos modelos de negócios, porém, os caminhos para que isso ocorra ainda é incerto. Os parâmetros 


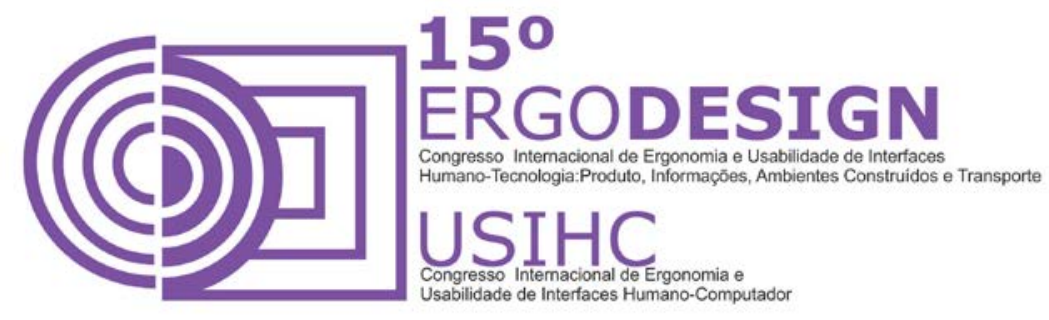

para se produzir um jornal diário também mudaram bastante, já que a notícia é veiculada no momento em que o fato ocorre. Notícia também é um outro termo passível de transformação, já que a democratização da informação, muito impulsionada pelas redes sociais, descentraliza-a de uma redação de jornal.

Independente dos rumos que tomarão as empresas veiculadoras de notícias é imprescindível entender como o usuário de notícias está obtendo essa informação, para que se projete novos modelos de negócios a partir do comportamento e da necessidade desses usuários.

\section{REFERÊNCIAS BIBLIOGRÁFICAS}

EVERLING, Marli Teresinha. Diretrizes para um ambiente de aprendizagem assíncrona no curso de design. 2011. 411 p. Tese de Doutorado - Departamento de Artes e Design, Pontifícia Universidade Católica Rio de Janeiro, Rio de Janeiro, 2011.

FLOOD, Robert Louis. The Relationship of 'Systems Thinking' to Action. Springer Science+Business Media, LLC, mar. 2010. Volume 23, 269-284 p.

Disponível em: < http://link.springer.com/article/10.1007\%2Fs11213-010-9169-1> Acesso em: 21 dez 2014.

FRISONI, Bianka Cappucci. Ergodesign, metodologia ergonômica, "designing" para o uso humano. 2000. 340 p. Dissertação de Mestrado - Departamento de Artes e Design, Pontifícia Universidade Católica do Rio de Janeiro, Rio de Janeiro, 2000.

LINSTONE, Harold A., TUROFF, Murray (Editores). The Delphi Method. Addison-Wesley Publishing Company, Massachussets, EUA, 1975

MORVILLE, Peter. Intertwingled: Information Changes Everything. 2014.

THE NEW YORK TIMES. Executive Sumary. New York, 2014. 97p. Relatório Técnico. 